Commun. Fac. Sci. Univ. Ank. Ser. A1 Math. Stat.

Volume 68, Number 2, Pages 20642078(2019)

DOI: $10.31801 /$ cfsuasmas. 586082

ISSN 1303-5991 E-ISSN 2618-6470

http://communications.science.ankara.edu.tr/index.php?series=A1

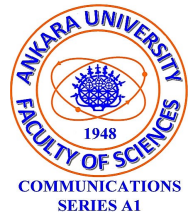

\title{
ALGORITHMIC APPROACH OF CORDIAL LABELING ON CARTESIAN PRODUCT BETWEEN BALANCED BIPARTITE
} GRAPH AND PATH

\author{
SUMONTA GHOSH AND ANITA PAL
}

\begin{abstract}
Cordial labeling is one of the useful labeling in the realm of graph labeling. For the graph $G=(V, E)$ vertex set $V$ is label by $\{0,1\}$, where labeling of edge set $E$ calculated by absolute difference between the end vertices labeling with the restriction that number of vertices label by 0 and number of vertices label by 1 , their absolute difference not exceeding 1 . If similar restriction will follow for edge also then the labeling technique is known as cordial labeling and the graph which admits cordial labeling is known as cordial graph. In this paper we want to apply cordial labeling on a complex graph structure which we obtained by Cartesian product between complete bipartite graph(Balanced bipartite graph) $K_{n, n}$ and path $P_{r}$. We also propose three algorithm to label the above said graph by cordial labeling. We have analyse the time complexity of each algorithm and which is super-linear.
\end{abstract}

\section{INTRODUCTION}

We are aware about graph labeling as it has acquired by many technology in real world. Josef Gallian [1] provide a survey paper on graph labeling which help us to investigate the research direction of graph labeling. Hegde [2] simplify the idea of graph labeling, where Beineke and Hegde [3] together state that graph labeling is a combination between number theory and graphs structure.

Ibrahim Cahit [4, 5, 6, 7, 8, 9, 10, 11, 12, 13, 14, worked on graceful [16] and harmonious [15] labelings. Cahit [5] paid his attention on some simple graph like tree, $K_{n}, K_{m, n}$, friendship graph, fans (with some limitation) and he proves that these graphs are cordial.

Received by the editors: February 05, 2018; Accepted: June 27, 2019.

2010 Mathematics Subject Classification. 05C15.

Key words and phrases. Cordial labeling, graph labeling, Cartesian product of graphs.

Submitted via International Conference on Current Scenario in Pure and Applied Mathematics [ICCSPAM 2018].

(C)2019 Ankara University Communications Faculty of Sciences University of Ankara-Series A1 Mathematics and Statistics 
Definition 1. Let $G=(V, E)$ be a graph with vertex set $V$ and edge set $E$ and the vertex set is label by $\{0,1\}$, where edge labeling calculated by absolute difference between the end vertices labeling with the restriction that number of vertices label by 0 and number of vertices label by 1 , their absolute difference not exceeding 1. If similar restriction will follow for edge also then the labeling technique is known as cordial labeling and the graph which admits cordial labeling is known as cordial graph.

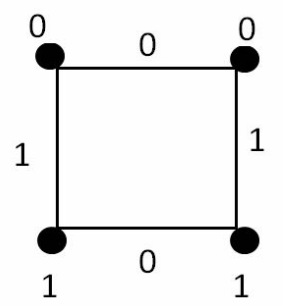

Figure 1. Cordial labeling.

The idea of cordial language and cordial numbers presented by Babujee and Shobana [17. There also exists some other types of labeling like product cordial labeling, total signed product cordial labeling and signed product cordial labeling. The concept of signed product cordial labeling was introduced by J. Baskar Babujee [17 and he proved that many graphs admit signed product cordial labeling. This idea is investigated by numerous researcher like P.P. Ulaganathanet [18, Santhi. M [20] and Saanthi. M et. al [19]. The vast majority of the analysts give careful consideration of cordial labeling on straightforward system and additionally complex system. We give a brief of the definitions and other data which are valuable for the present examination.

Definition 2. Cartesian product of two graphs $G=(V, E)$ and $H=\left(V^{\prime}, E^{\prime}\right)$ is the Cartesian product between two arrangement of vertices $V(G) \times V^{\prime}(H)$ denoted by $G \times H$, where $\left(u, u^{\prime}\right)$ and $\left(v, v^{\prime}\right)$ are the order pair of the Cartesian product will be adjoining in $G \times H$ if and just assuming either

(1) $u=v$ and $u^{\prime}$ is adjacent with $v^{\prime}$ in $H$, or

(2) $u^{\prime}=v^{\prime}$ and $u$ is adjacent with $v$ in $G$.

The Cartesian product of two graphs are commutative.

As time goes usage of frameworks turn out to be high, which experienced more extensive and complex system structure. Association of various kinds of system demonstrate assumes indispensable part, all things considered, so result of two existing system display gives a complex system structure with the advantage of single coordinated system. Such kind of complex system may prompt high cost 


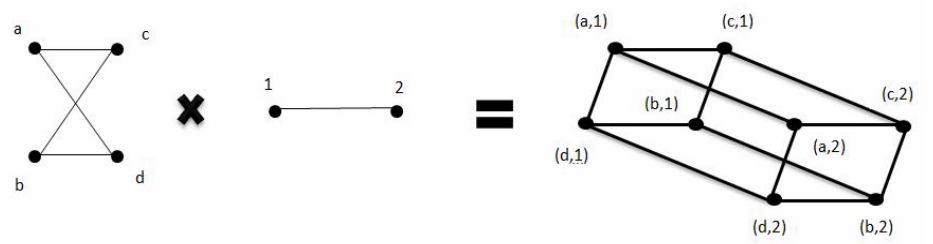

Figure 2. Cartesian product of two graphs

factor in correspondence yet it encountered a high dependability moreover. In this paper, we mainly concentrate on Cartesian product between $K_{n, n} \times P_{r}$.

Whatever is left of the paper is composed is as per the following. Section 2 contains some preliminaries and definitions, Section 3 presents algorithms to label Cartesian product between $K_{n, n} \times P_{r}$, analysis of algorithm followed by conclusion.

\section{Preliminaries}

Definition 3. A graph $G=(V, E)$ is called a complete bipartite graph if its vertices can be clustered into two subsets $V_{1}$ and $V_{2}$, where there exist no edges in the same subset, and each vertex of $V_{1}\left(V_{2}\right)$ is connected with all vertices of $V_{2}\left(V_{1}\right)$. Here $V_{1}=\left\{X_{11}, X_{12}, \ldots, X_{1 m}\right\}$ contains $m$ vertices and $V_{2}=\left\{Y_{11}, Y_{12}, \ldots, Y_{1 n}\right\}$ contains $n$ vertices.

A complete bipartite graph with $\left|V_{1}\right|=m$ and $\left|V_{2}\right|=n$ is denoted by $K_{m, n}$. When $\left|V_{1}\right|=\left|V_{2}\right|$ then complete bipartite graph is known as balanced bipartite graph and which is denoted by $K_{n, n}$.

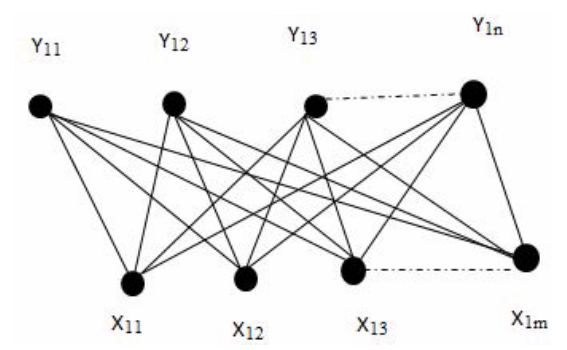

Figure 3. Complete bipartite graph $K_{m, n}$

The Cartesian product $K_{n, n} \times P_{r}$ can be visualized in a simple way. For this product, we draw $r$ copies of $K_{n, n}$. Let $X_{i}=\left\{x_{i 1}, x_{i 2}, x_{i 3}, \ldots, x_{i p}, \ldots, x_{i n}\right\}$ and $Y_{j}=\left\{y_{j 1}, y_{j 2}, y_{j 3}, \ldots, y_{j q}, \ldots, y_{j n}\right\}$ be the set of vertices of the $i$ th copy of the graph 


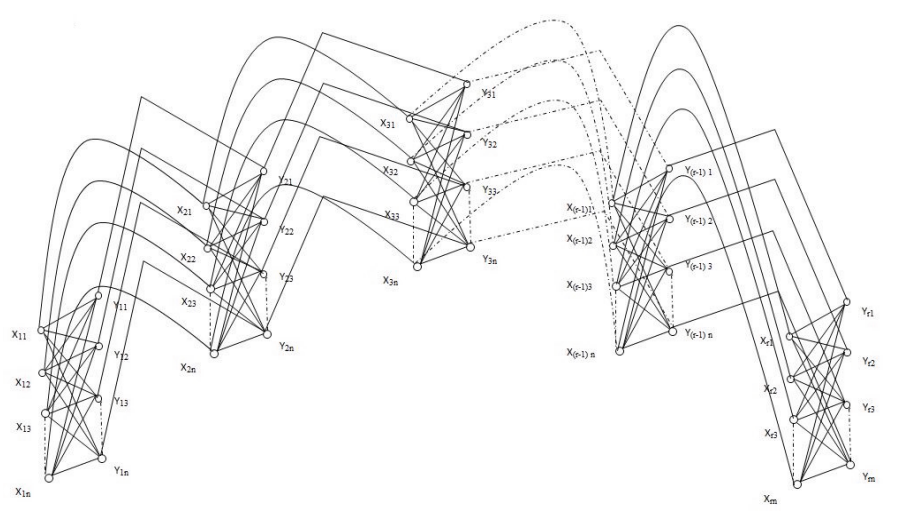

FiguRE 4. The graph $K_{n, n} \times P_{r}$

$K_{n, n}$. The vertices of $i$ th copy of $K_{n, n}$ are connected with $(i+1)$ th copy of $K_{n, n}$ only as per following rule:

(1) $x_{i_{1} p_{1}}$ and $x_{i_{2} p_{2}}$ will be connected if $p_{1}=p_{2}$ and $\left|i_{1}-i_{2}\right|=1$

(2) $y_{j_{1} q_{1}}$ and $y_{j_{2} q_{2}}$ will be connected if $q_{1}=q_{2}$ and $\left|j_{1}-j_{2}\right|=1$

Note that the set of vertices of $G=(V, E)=K_{n, n} \times P_{r}$ is $\cup_{i=1}^{n} X_{i} \cup_{j=1}^{n} Y_{j}$. It is clear that $\left(x_{i p}, y_{j q}\right) \in E$, i.e $d\left(x_{i p}, y_{j q}\right)=1$ for $i, j=1,2,3, \ldots, r$ and $p, q=$ $1,2,3, \ldots, n$. Again $d\left(x_{i p}, x_{(i+1) p}\right)=1$ for $i=1,2,3, \ldots, r, p=1,2,3, \ldots, n$ and $d\left(y_{j q}, y_{(j+1) q}\right)=1$ for $j=1,2,3, \ldots, r, q=1,2,3, \ldots, n$.

To obtained the graph $G=K_{n, n} \times C_{r}$, graph $K_{n, n}$ has to copy $r$ times. Here each $K_{n, n}$ has two set of vertices $X, Y$ where $|X|=n$ and $|Y|=n$. Each set of vertices of $K_{n, n}$ for $r$ copies represented by $\left(X_{1}, Y_{1}\right),\left(X_{2}, Y_{2}\right),\left(X_{3}, Y_{3}\right), \ldots,\left(X_{r}, Y_{r}\right)$, where each $X_{i}=\left\{x_{i 1}, x_{i 2}, x_{i 3}, \ldots, x_{i n}\right\}$ and $Y_{i}=\left\{y_{i 1}, y_{i 2}, y_{i 3}, \ldots, y_{i n}\right\}$.

Consider the graph $G=\left(K_{n, n} \times C_{r}\right)$, where $V=\bigcup_{i=1}^{r} v_{i}$ and $v_{i}=\left(X_{i}, Y_{i}\right)$, where $X_{i}=\left\{x_{i 1}, x_{i 2}, x_{i 3}, \ldots, x_{i n}\right\}$ and $Y_{i}=\left\{y_{i 1}, y_{i 2}, y_{i 3}, \ldots, y_{i n}\right\}$ for each $i=1,2,3, \ldots, r$. Two vertices connected by the following way.

(1) $x_{i_{1} j}$ and $x_{i_{2} k}$ will be connected if $j=k$ and $\left|i_{1}-i_{2}\right|=1$ or $(r-1)$

(2) $y_{i_{1} p}$ and $y_{i_{2} q}$ will be connected if $p=q$ and $\left|i_{1}-i_{2}\right|=1$ or $(r-1)$

and it is clear that $\left(x_{i j}, y_{i p}\right) \in E$, i.e $d\left(x_{i j}, y_{i p}\right)=1$ for $i=1,2,3, \ldots, r, j=$ $1,2,3, \ldots, n$ and $p=1,2,3, \ldots, n$. Again $d\left(x_{i j}, x_{(i+1) j}\right)=1$ for $i=1,2,3, \ldots, r$, $j=1,2,3, \ldots, n$ and $d\left(y_{i p}, y_{(i+1) p}\right)=1$ for $i=1,2,3, \ldots, r, p=1,2,3, \ldots, n$. 


\section{Labeling Scheme of Cartesian Product Between Balanced Bipartite Graph and Path}

3.1. Algorithm. Now we are going to label the graph $G=K_{n, n} \times P_{r}$, let us take a balanced bipartite graph $K_{n, n}$. As we know that each $K_{n, n}$ having two set of vertices $X_{i}$ and $Y_{i}$ for $i=1,2,3, \ldots, r$ and $\left|X_{i}\right|=\left|Y_{i}\right|=n$ where the vertices connected according to the rule of complete bipartite graph. So, $X_{i}$ and $Y_{i}$ both has $n$ vertices where $X_{i}=\left\{x_{i 1}, x_{i 2}, x_{i 3}, \ldots x_{i j}, \ldots, x_{i n}\right\}$ similarly $Y_{i}=\left\{y_{i 1}, y_{i 2}, y_{i 3}, \ldots, y_{i j}, \ldots, y_{i n}\right\}$.

To label the graph $G=K_{n, n} \times P_{r}$ we have design four algorithms. Algorithm 4 is for cordial labeling of $G=K_{n, n}$ where proposed Algorithm 6 design for the graph $G=K_{n, n} \times P_{3}$, Algorithm 8 and Algorithm 10 are for $G=K_{n, n} \times P_{r}$ where $n$ is odd and $r>3$ and $G=K_{n, n} \times P_{r}$ where $n$ is even. To obtained the graph $G=K_{n, n} \times P_{r}$ we need to draw $r$ copies of $K_{n, n}$. As Algorithm 8 is working for path length $r>3$ so for the graph $G=K_{n, n} \times P_{r}$ after labeling up to multiple of 4 copies of $K_{n, n}$ remaining copies of $K_{n, n}$ will follow Algorithm 6. Through out this paper we denote $V_{0}(G)$ and $V_{1}(G)$ are the set of vertices label by 0 and 1 respectively, similarly denote $E_{0}(G)$ and $E_{1}(G)$ are the set of edges label by 0 and 1 respectively

Labeling of the vertex $v_{i}$ is denoted by $f\left(v_{i}\right)$ for each algorithm. According to the pattern of Cartesian product between $K_{n, n}$ and $P_{r}$ we have $r$ copies of $K_{n, n}$. We have to label each copy of $K_{n, n}$ by 0 or 1 .

\subsection{Algorithm.}

Algorithm 4. Cordial labeling of $K_{n, n}(C L B B)$

Input: $G=K_{n, n}$.

Output: Labelled graph $G=K_{n, n}$.

Step 1 loop $j=1$ to $n$

Step 2

(1) Assign label $f\left(x_{1 j}\right)=0$ if $(1+j)$ divisible by 2 .

(2) Assign label $f\left(y_{1 j}\right)=1$ if $(1+j)$ divisible by 2 .

\section{Step 3}

(1) Assign label $f\left(x_{1 j}\right)=1$ if $(1+j)$ not divisible by 2 .

(2) Assign label $f\left(y_{1 j}\right)=0$ if $(1+j)$ not divisible by 2 .

end of loop $j$.

Stop.

3.2.1. The proof of correctness of the algorithm 4

Theorem 5. Algorithm 4 proof that the graph $K_{n, n}$ is a cordial graph. 
Proof. Let us consider the graph $G=K_{n, n}$, where $V(G)$ has has the cardinality $2 n$ and $E(G)$ has the cardinality $n^{2}$. From algorithm 4 we can investigate that $\left|V_{0}(G)\right|=n$ and $\left|V_{1}(G)\right|=n$. So ||$V_{0}(G)|-| V_{1}(G)||=|n-n|=0 \leq 1$. Now for satisfying edge labeling condition we consider two cases.

Case 1: When $n$ is even.

When $n$ is even it is clear that $\left|E_{0}(G)\right|=\frac{n^{2}}{2}$ and $\left|E_{1}(G)\right|=\frac{n^{2}}{2}$. So ||$E_{0}(G) \mid-$ $\left|E_{1}(G)\right||=| \frac{n^{2}}{2}-\frac{n^{2}}{2} \mid=0 \leq 1$.

Case 2: When $n$ is odd.

When $n$ is odd it is clear that $\left|E_{0}(G)\right|=\frac{\left(n^{2}-1\right)}{2}$ and $\left|E_{1}(G)\right|=\frac{\left(n^{2}+1\right)}{2}$. So ||$E_{0}(G) \mid-$ $\left|E_{1}(G)\right||=| \frac{\left(n^{2}-1\right)}{2}-\frac{\left(n^{2}+1\right)}{2} \mid=1 \leq 1$.

The graph $G=K_{n, n}$ follow the property of cordial labeling, so the graph $G=K_{n, n}$ is cordial graph.

3.2.2. Analysis of Algorithm 4. As an example let us consider a balanced bipartite graph $G=K_{5,5}$ with the set of vertices $X=\left\{x_{11}, x_{12}, x_{13}, x_{14}, x_{15}\right\}$ and $Y=\left\{y_{11}, y_{12}, y_{13}, y_{14}, y_{15}\right\}$. Now we can assign the value to the vertices for the value of $j=1,2,3,4,5$. We can check that $(1+1)$ is divisible by 2 , so put $f\left(x_{11}\right)=0$ and $f\left(y_{11}\right)=1$ and $(1+2)$ is not divisible by 2 , so put $f\left(x_{12}\right)=1$ and $f\left(y_{12}\right)=0$ similarly $f\left(x_{13}\right)=0, f\left(y_{13}\right)=1, f\left(x_{14}\right)=1, f\left(y_{14}\right)=0, f\left(x_{15}\right)=0$ and $f\left(x_{15}\right)=1$. So, $\left|V_{0}(G)\right|=5$ and $\left|V_{1}(G)\right|=5$ which is differ by 0 and $\left|E_{0}(G)\right|=12$ and $\left|E_{1}(G)\right|=13$ which is differ by 1 .

We also analyze the time complexity of the above algorithm which is linear and $\bigcirc(n)$.

TABLE 1. Vertex label and Edge label matrix of $K_{5,5}$

$\begin{array}{lllllll}0 & 1 & 1 & 0 & 1 & 0 & 1 \\ 1 & 0 & 0 & 1 & 0 & 1 & 0 \\ 0 & 1 & 1 & 0 & 1 & 0 & 1 \\ 1 & 0 & 0 & 1 & 0 & 1 & 0 \\ 0 & 1 & 1 & 0 & 1 & 0 & 1\end{array}$


3.3. Algorithm. This algorithm is design for maximum path length 3 but by restricting the loop we are able to consider path length 2 also.

Algorithm 6 (h). Cordial labeling of cartesian product between $K_{n, n}$ and $P_{3}$ (CLBBP3) Input: $G=\left(K_{n, n} \times P_{3}\right)$, where $n$ is odd.

Output: Labelled graph $G=\left(K_{n, n} \times P_{3}\right)$.

Step 1 loop $i=1$ to 3

Step 2 if $i$ divisible by 2 then continue

Step 3 loop $j=1$ to $n$

(1) Assign label $f\left(x_{i j}\right)=0$ if $(i+j)$ divisible by 2 .

(2) Assign label $f\left(y_{i j}\right)=1$ if $(i+j)$ divisible by 2 .

and

(1) Assign label $f\left(x_{i j}\right)=1$ if $(i+j)$ not divisible by 2 .

(2) Assign label $f\left(y_{i j}\right)=0$ if $(i+j)$ not divisible by 2 .

end of loop $j$.

Step 4 if $i$ divisible by 2 then continue

Step 5 loop $j=1$ to $(n-1)$

(1) Assign label $f\left(x_{i j}\right)=1$ if $(i+j)$ divisible by 2 .

(2) Assign label $f\left(y_{i j}\right)=1$ if $(i+j)$ divisible by 2 .

and

(1) Assign label $f\left(x_{i j}\right)=0$ if $(i+j)$ not divisible by 2 .

(2) Assign label $f\left(y_{i j}\right)=0$ if $(i+j)$ not divisible by 2 .

end of loop $j$.

Step 6 Assign label $f\left(x_{i n}\right)=0$ and $f\left(y_{i n}\right)=1$ (labeling of $n^{\text {th }}$ vertices)

end of loop $i$.

Stop.

3.3.1. The proof of correctness of the algorithm 6 .

Theorem 7. Algorithm 6 proof the graph the graph $K_{n, n} \times P_{3}$ is cordial graph.

Proof. Algorithm 6 work for the graph $G=K_{n, n} \times P_{3}$ when $n$ is odd, where $V(G)$ has cardinality $6 n$ and $E(G)$ has the cardinality $3 n^{2}+4 n$. By following the algorithm 6 we get $\left|V_{0}(G)\right|=3 n$ and $\left|V_{1}(G)\right|=3 n$. So ||$V_{0}(G)|-| V_{1}(G)||=|3 n-3 n|=$ $0 \leq 1$. We get edge label as $\left|E_{0}(G)\right|=\frac{\left(3 n^{2}+4 n+1\right)}{2}$ and $\left|E_{1}(G)\right|=\frac{\left(3 n^{2}+4 n-1\right)}{2}$, i.e ||$E_{0}(G)|-| E_{1}(G)||=\left|\frac{\left(3 n^{2}+4 n-1\right)}{2}-\frac{\left(3 n^{2}+4 n+1\right)}{2}\right|=1 \leq 1$. The graph $G=K_{n, n} \times P_{3}$ follow the property of cordial labeling, so the graph $G=K_{n, n} \times P_{3}$ is cordial graph.

3.3.2. Analysis of Algorithm CLBBP3. For example of the Algorithm 6 we consider the graph $G=K_{5,5} \times P_{3}$, for each copies of $K_{5,5}$ we have $X_{i}=\left\{x_{i 1}, x_{i 2}, \ldots, x_{i j}, \ldots, x_{i 5}\right\}$ and $Y_{i}=\left\{y_{i 1}, y_{i 2}, \ldots, y_{i j}, \ldots, y_{i 5}\right\}$ for $i=1,2,3$. As we have three $K_{5,5}$, for $i=1$ and 
$i$ is not divisible by 2 , for each values of $j=1,2,3,4,5$ assign $f\left(x_{11}\right)=0, f\left(y_{11}\right)=1$ as $(1+1)$ is divisible by 2 and $f\left(x_{12}\right)=1, f\left(y_{12}\right)=0$ as $(1+2)$ is not divisible by 2 similarly $f\left(x_{13}\right)=0, f\left(y_{13}\right)=1, f\left(x_{14}\right)=1, f\left(y_{14}\right)=0, f\left(x_{15}\right)=0, f\left(y_{15}\right)=1$. Now for $i=2$ checked and clear that $i$ is divisible by 2 , for each values of $j=1,2,3,4$ assign $f\left(x_{21}\right)=0, f\left(y_{21}\right)=0$ as $(2+1)$ is not divisible by 2 and $f\left(x_{22}\right)=1$, $f\left(y_{22}\right)=1$ as $(2+2)$ is divisible by 2 , similarly $f\left(x_{23}\right)=0, f\left(y_{23}\right)=0, f\left(x_{24}\right)=1$, $f\left(y_{24}\right)=1$ and for the pair of vertices $f\left(x_{25}\right)=0, f\left(y_{25}\right)=1$. For $i=3$, as $i$ is not divisible by 2 it will follow the same procedure as $i=1$.

We can check $\left|V_{0}(G)\right|=15$ and $\left|V_{1}(G)\right|=15$, which differ by 0 . Edge labeling $\left|E_{0}(G)\right|=48$ and $\left|E_{1}(G)\right|=47$, which is differ by 1 , so imposed rule of cordial labeling is worked successfully. We also analyze the time complexity of the above algorithm which is linear and $\bigcirc(3 n)$.

TABLE 2. Vertex label matrix of $K_{5,5} \times P_{3}$

$\begin{array}{llllll}0 & 1 & 0 & 0 & 0 & 1 \\ 1 & 0 & 1 & 1 & 1 & 0 \\ 0 & 1 & 0 & 0 & 0 & 1 \\ 1 & 0 & 1 & 1 & 1 & 0 \\ 0 & 1 & 0 & 1 & 0 & 1\end{array}$

TABLE 3. Edge label matrix of $K_{5,5} \times P_{3}$

$\begin{array}{lllllllllllllllllll}1 & 0 & 1 & 0 & 1 & 0 & 1 & 0 & 1 & 0 & 1 & 1 & 0 & 1 & 1 & 0 & 1 & 0 & 1 \\ 0 & 1 & 0 & 1 & 0 & 0 & 1 & 1 & 0 & 1 & 0 & 0 & 0 & 1 & 0 & 1 & 0 & 1 & 0 \\ 1 & 0 & 1 & 0 & 1 & 0 & 1 & 0 & 1 & 0 & 1 & 1 & 0 & 1 & 1 & 0 & 1 & 0 & 1 \\ 0 & 1 & 0 & 1 & 0 & 0 & 1 & 1 & 0 & 1 & 0 & 0 & 0 & 1 & 0 & 1 & 0 & 1 & 0 \\ 1 & 0 & 1 & 0 & 1 & 0 & 0 & 0 & 1 & 0 & 1 & 1 & 0 & 0 & 1 & 0 & 1 & 0 & 1\end{array}$




\subsection{Algorithm.}

Algorithm 8. Cordial labeling of cartesian product between $K_{n, n}$ and $P_{r} C L B B P(O)$

We design this algorithm for the graph $G=K_{n, n} \times P_{r}$ where $n$ is odd and $r>3$.

For shake of our algorithm we calculate $r \equiv q(\bmod 4)$ and $t=(r-q)$. Input: The graph $G=\left(K_{n, n} \times P_{r}\right)$.

Output: Labelled graph $G=\left(K_{n, n} \times P_{r}\right)$.

Step 1 loop $i=1$ to $t$

Step 2 if $i$ is not divisible by 2 then

Step 3 loop $j=1$ to $(n-1)$

(1) Assign label $f\left(x_{i j}\right)=0$ if $(i+j)$ divisible by 2.

(2) Assign label $f\left(y_{i j}\right)=1$ if $(i+j)$ divisible by 2 .

and

(1) Assign label $f\left(x_{i j}\right)=1$ if $(i+j)$ not divisible by 2 .

(2) Assign label $f\left(y_{i j}\right)=0$ if $(i+j)$ not divisible by 2 .

end of loop $j$.

Step 4 Assign label $f\left(x_{i n}\right)=0$ and $f\left(y_{i n}\right)=1$.

Step 5 else if $i$ is divisible by 4 then

Step 6 loop $j=1$ to $n$

(1) Assign label $f\left(x_{i j}\right)=1$ if $(i+j)$ divisible by 2 .

(2) Assign label $f\left(y_{i j}\right)=1$ if $(i+j)$ divisible by 2 .

and

(1) Assign label $f\left(x_{i j}\right)=0$ if $(i+j)$ not divisible by 2 .

(2) Assign label $f\left(y_{i j}\right)=0$ if $(i+j)$ not divisible by 2 .

end of loop $j$.

Step 7 else

Step 8 loop $j=1$ to $n$

(1) Assign label $f\left(x_{i j}\right)=0$ if $(i+j)$ divisible by 2 .

(2) Assign label $f\left(y_{i j}\right)=0$ if $(i+j)$ divisible by 2 .

and

(1) Assign label $f\left(x_{i j}\right)=1$ if $(i+j)$ not divisible by 2 .

(2) Assign label $f\left(y_{i j}\right)=1$ if $(i+j)$ not divisible by 2 .

end of loop $j$.

end of loop $i$.

Step 9 For remaining $K_{n, n}$, we follow algorithn 6

Stop.

3.4.1. The proof of correctness of the algorithm 8 .

Theorem 9. Algorithm 8 proof that the graph $G=K_{n, n} \times P_{r}$ (where $n$ is odd) is cordial graph. 
Proof. To prove Algorithm 8 we consider the graph $G=K_{n, n} \times P_{r}$ when $n$ is odd, where $V(G)$ has the cardinality $2 n r$ and $E(G)$ has the cardinality $n^{2} r+2 n r-2 n$. According to Algorithm 8 we get $\left|V_{0}(G)\right|=n r$ and $\left|V_{1}(G)\right|=n r$, so ||$V_{0}(G) \mid-$ $\left|V_{1}(G)\right||=| n r-n r \mid=0 \leq 1$. Now for satisfying edge labelling condition we consider two cases.

Case 1: When $r$ is odd When $r$ is odd, we have $\left|E_{0}(G)\right|=\frac{\left(n^{2} r+2 n r-2 n-1\right)}{2}$ and $\left|E_{1}(G)\right|=\frac{\left(n^{2} r+2 n r-2 n+1\right)}{2}$, i.e.

$$
|| E_{0}(G)|-| E_{1}(G)||=\left|\frac{\left(n^{2} r+2 n r-2 n-1\right)}{2}-\frac{\left(n^{2} r+2 n r-2 n+1\right)}{2}\right|=1 \leq 1 .
$$

Case 2: When $r$ is even When $r$ is even, $\left|E_{0}(G)\right|=\frac{\left(n^{2} r+2 n r-2 n\right)}{2}$ and $\left|E_{1}(G)\right|=$ $\frac{\left(n^{2} r+2 n r-2 n\right)}{2}$, i.e.

$$
|| E_{0}(G)|-| E_{1}(G)||=\left|\frac{\left(n^{2} r+2 n r-2 n\right)}{2}-\frac{\left(n^{2} r+2 n r-2 n\right)}{2}\right|=0 \leq 1 .
$$

The graph $G=K_{n, n} \times P_{r}$ follow the property of cordial labeling, so the graph $G=K_{n, n} \times P_{r}$ is cordial graph.

3.4.2. Analysis of Algorithm 8, For analysis of the Algorithm 8 we consider the graph $G=K_{5,5} \times P_{7}$, for each copies of $K_{5,5}$ we have $X_{i}=\left\{x_{i 1}, x_{i 2}, \ldots, x_{i j}, \ldots, x_{i 5}\right\}$ and $Y_{i}=\left\{y_{i 1}, y_{i 2}, \ldots, y_{i j}, \ldots, y_{i 5}\right\}$ for $i=1,2,3,4,5,6,7$. Now we have seven copies of $K_{5,5}$ as the path length is $r=7$, so calculate $7 \equiv q(\bmod 4)$ and the value of $q=3$ and $t=(7-3)=4$ so we can label up to 4 copies of $k_{5,5}$, for the rest 3 copies of $K_{5,5}$ we call the Algorithm 6. According to above algorithm loop of $i$ will run up to 4 and for $i=1$ as $i$ is odd, for each values of $j=1,2,3,4,5$ assign $f\left(x_{11}\right)=0$, $f\left(y_{11}\right)=1$ as $(1+1)$ is divisible by 2 and $f\left(x_{12}\right)=1, f\left(y_{12}\right)=0$ as $(1+2)$ is not divisible by 2 . similarly $f\left(x_{13}\right)=0, f\left(y_{13}\right)=1, f\left(x_{14}\right)=1, f\left(y_{14}\right)=0, f\left(x_{15}\right)=0$, $f\left(y_{15}\right)=1$. Now for $i=2$ as $i$ is divisible by 2 , for each values of $j=1,2,3,4$ assign $f\left(x_{21}\right)=1, f\left(y_{21}\right)=1$ as $(2+1)$ is not divisible by 2 and $f\left(x_{22}\right)=0, f\left(y_{22}\right)=0$ as as $(2+2)$ is divisible by 2 , similarly $f\left(x_{23}\right)=1, f\left(y_{23}\right)=1, f\left(x_{24}\right)=0, f\left(y_{24}\right)=0$ and for the last pair of vertices $f\left(x_{25}\right)=0, f\left(y_{25}\right)=1$. For $i=3$ as $i$ is odd, it will follow the same procedure as $i=1$. When the value of $i=4$ as $i$ is divisible by 4 , for each values of $j=1,2,3,4,5$ assign $f\left(x_{41}\right)=0, f\left(y_{41}\right)=0, f\left(x_{42}\right)=1$, $f\left(y_{42}\right)=1, f\left(x_{43}\right)=0, f\left(y_{43}\right)=0, f\left(x_{44}\right)=1, f\left(y_{44}\right)=1, f\left(x_{45}\right)=0, f\left(y_{45}\right)=0$. For the last three copies of $K_{5,5}$ we call Algorithm 6 .

We can check $\left|V_{0}(G)\right|=35$ and $\left|V_{1}(G)\right|=35$, which differ by 0 . Edge labeling $\left|E_{0}(G)\right|=117$ and $\left|E_{1}(G)\right|=118$, which is differ by 1 , so imposed rule of cordial labeling is worked successfully. We also analyze the time complexity of the above algorithm which is super linear and it is $\bigcirc(n r)$. 
TABle 4. Vertex label matrix of $K_{5,5} \times P_{7}$

$\begin{array}{llllllllllllll}0 & 1 & 1 & 1 & 0 & 1 & 0 & 0 & 0 & 1 & 0 & 0 & 0 & 1 \\ 1 & 0 & 0 & 0 & 1 & 0 & 1 & 1 & 1 & 0 & 1 & 1 & 1 & 0 \\ 0 & 1 & 1 & 1 & 0 & 1 & 0 & 0 & 0 & 1 & 0 & 0 & 0 & 1 \\ 1 & 0 & 0 & 0 & 1 & 0 & 1 & 1 & 1 & 0 & 1 & 1 & 1 & 0 \\ 0 & 1 & 1 & 1 & 0 & 1 & 0 & 0 & 0 & 1 & 0 & 1 & 0 & 1\end{array}$

TABLE 5. Edge label matrix of $K_{5,5} \times P_{7}$

$\begin{array}{llllllllllllll}1 & 0 & 1 & 0 & 1 & 1 & 0 & 0 & 1 & 0 & 1 & 0 & 1 & 0 \\ 0 & 1 & 0 & 1 & 0 & 1 & 0 & 1 & 0 & 1 & 0 & 1 & 1 & 0 \\ 1 & 0 & 1 & 0 & 1 & 1 & 0 & 0 & 1 & 0 & 1 & 0 & 1 & 0 \\ 0 & 1 & 0 & 1 & 0 & 1 & 0 & 1 & 0 & 1 & 0 & 1 & 1 & 0 \\ 1 & 0 & 1 & 0 & 1 & 1 & 0 & 0 & 1 & 0 & 1 & 0 & 1 & 0\end{array}$

$\begin{array}{llllllllllllll}1 & 0 & 1 & 0 & 1 & 0 & 1 & 0 & 1 & 0 & 1 & 0 & 0 & 1 \\ 0 & 1 & 0 & 1 & 0 & 0 & 1 & 1 & 0 & 1 & 0 & 1 & 0 & 1 \\ 1 & 0 & 1 & 0 & 1 & 0 & 1 & 0 & 1 & 0 & 1 & 0 & 0 & 1 \\ 0 & 1 & 0 & 1 & 0 & 0 & 1 & 1 & 0 & 1 & 0 & 1 & 0 & 1 \\ 1 & 0 & 1 & 0 & 1 & 0 & 1 & 0 & 1 & 0 & 1 & 0 & 0 & 1\end{array}$

$\begin{array}{lllllllllllllllllll}1 & 0 & 1 & 0 & 1 & 0 & 1 & 0 & 1 & 0 & 1 & 1 & 0 & 1 & 1 & 0 & 1 & 0 & 1 \\ 0 & 1 & 0 & 1 & 0 & 0 & 1 & 1 & 0 & 1 & 0 & 0 & 0 & 1 & 0 & 1 & 0 & 1 & 0 \\ 1 & 0 & 1 & 0 & 1 & 0 & 1 & 0 & 1 & 0 & 1 & 1 & 0 & 1 & 1 & 0 & 1 & 0 & 1 \\ 0 & 1 & 0 & 1 & 0 & 0 & 1 & 1 & 0 & 1 & 0 & 0 & 0 & 1 & 0 & 1 & 0 & 1 & 0 \\ 1 & 0 & 1 & 0 & 1 & 0 & 0 & 0 & 1 & 0 & 1 & 1 & 0 & 0 & 1 & 0 & 1 & 0 & 1\end{array}$

3.5. Algorithm. This algorithm we have design for the graph $G=\left(K_{n, n} \times P_{r}\right)$, for even $n$.

Algorithm 10. Cordial labeling of cartesian product between $K_{n, n}$ and $P_{r} C L B B P(E)$ Input: A Cartesian product between $K_{n, n}$ and $P_{r}$ i.e $G=\left(K_{n, n} \times P_{r}\right)$, where $n$ is even.

Output: Cordial labeling of the graph $G=\left(K_{n, n} \times P_{r}\right)$.

Step 1 loop $i=1$ to $r$

Step 2 if $i=1$ is not divisible by 2 . loop $j=1$ to $n$

(1) $f\left(x_{i j}=1\right)$ if $(i+j)$ divisible by 2 . 
(2) $f\left(x_{i j}=0\right)$ if $(i+j)$ not divisible by 2 .

and

(1) $f\left(y_{i j}=0\right)$ if $(i+j)$ divisible by 2 .

(2) $f\left(y_{i j}=1\right)$ if $(i+j)$ not divisible by 2 .

end of loop.

Step 3 if $i$ is divisible by 2 then

loop $j=1$ to $n$

(1) $f\left(x_{i j}=0\right)$ if $(i+j)$ not divisible by 2 .

(2) $f\left(x_{i j}=1\right)$ if $(i+j)$ divisible by 2 .

and

(1) $f\left(y_{i j}=0\right)$ if $(i+j)$ not divisible by 2 .

(2) $f\left(y_{i j}=1\right)$ if $(i+j)$ divisible by 2 .

end of loop.

end of loop.

Stop.

3.5.1. The proof of correctness of the algorithm 10 given below.

Theorem 11. Algorithm 10 proof that the graph $G=K_{n, n} \times P_{r}$ (where $n$ is even) is cordial graph.

Proof. To prove Algorithm 10 we consider the graph $G=K_{n, n} \times P_{r}$ when $n$ is even, where $V(G)$ has the cardinality $2 n r$ and $E(G)$ has the cardinality $n^{2} r+2 n r-2 n$. According to Algorithm 10 we have $\left|V_{0}(G)\right|=n r$ and $\left|V_{1}(G)\right|=n r$, so ||$V_{0}(G) \mid-$ $\left|V_{1}(G)\right||=| n r-n r \mid=0 \leq 1$. For any value of $r$ we have the edge label $\left|E_{0}(G)\right|=$ $\frac{\left(n^{2} r+2 n r-2 n\right)}{2}$ and $\left|E_{1}(G)\right|=\frac{\left(n^{2} r+2 n r-2 n\right)}{2}$, so $\left|\frac{\left(n^{2} r+2 n r-2 n\right)}{2}-\frac{\left(n^{2} r+2 n r-2 n\right)}{2}\right|=0 \leq 1$. The graph $G=K_{n, n} \times P_{r}$ follow the property of cordial labeling, so the graph $G=K_{n, n} \times P_{r}$ is cordial graph.

3.5.2. Analysis of Algorithm 10. In our example we consider $G=K_{4,4} \times P_{4}$ and already there is 4 copies of $K_{4,4}$ with the vertex set $X_{i}$ and $Y_{i}$ for $i=1,2,3,4$. Now just considering the $1^{\text {st }}$ and $2^{\text {nd }}$ copy where $X_{1}=\left\{x_{11}, x_{12}, x_{13}, x_{14}\right\}$ and $X_{2}=\left\{x_{21}, x_{22}, x_{23}, x_{24}\right\}$. As we consider the $1^{\text {st }}$ copy so $i=1$ as $i$ is not divisible by 2 , for the values of $j=1,2,3,4$ corresponding label is $f\left(x_{11}\right)=f\left(x_{13}\right)=1$ as $(1+1)$ and $(1+3)$ both divisible by 2 and $f\left(x_{12}\right)=f\left(x_{14}\right)=0$ and $(1+2)$ and $(1+4)$ is not divisible by 2 . Now for the $2^{\text {nd }}$ copy $i=2$ as $i$ is divisible by 2 , for the values of $j=1,2,3,4$ and the corresponding label is $f\left(x_{21}\right)=f\left(x_{23}\right)=0$ as $(2+1)$ and $(2+3)$ is not divisible by 2 and $f\left(x_{22}\right)=f\left(x_{24}\right)=1$ as $(2+2)$ and $(2+4)$ is divisible by 2. For $Y_{1}=\left\{y_{11}, y_{12}, y_{13}, y_{14}\right\}$ and $Y_{2}=\left\{y_{21}, y_{22}, y_{23}, y_{24}\right\}$. As we consider the $1^{\text {st }}$ copy so $i=1$ as $i$ is odd and the corresponding label is $f\left(y_{11}\right)=f\left(y_{13}\right)=0$ as $(1+1)$ and $(1+3)$ is divisible by 2 and $f\left(y_{12}\right)=f\left(y_{14}\right)=1$ and $(1+2)$ and $(1+4)$ is not divisible by 2 . Now for the $2^{\text {nd }}$ copy $i=2$ as $i$ is even and the corresponding label is $f\left(y_{21}\right)=f\left(y_{23}\right)=0$ as $(2+1)$ and $(2+3)$ is not divisible by 2 and 


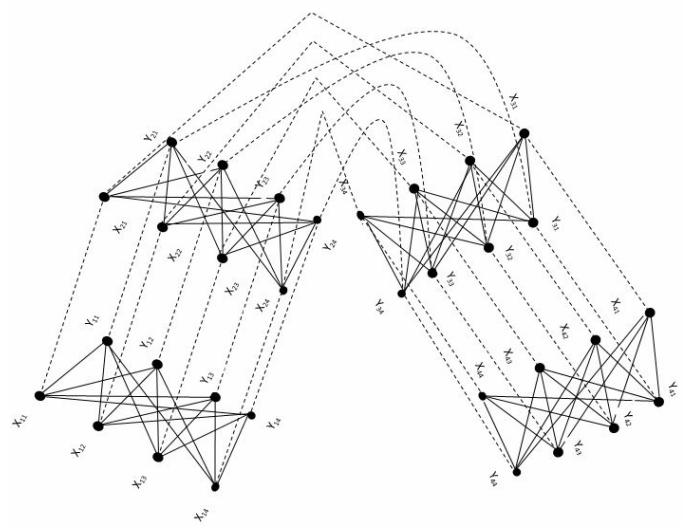

Figure 5. The graph $K_{4,4} \times P_{4}$

$f\left(y_{22}\right)=f\left(y_{24}\right)=1$ as $(2+2)$ and $(2+4)$. Rest of the two copy will follow the same rule to label by 0 and 1 . As we consider $K_{n, n}$ for all $n$ is even so we can observe that difference between the vertices label by 0 and the vertices label by 1 is 0 . We also give the edge label matrix which shows that the difference between edge label by 0 and the edge label by 1 is 0 also. Which follow the restriction of cordial labeling.

We can check $\left|V_{0}(G)\right|=16$ and $\left|V_{1}(G)\right|=16$, which differ by 0 . Edge labeling $\left|E_{0}(G)\right|=44$ and $\left|E_{1}(G)\right|=44$, which is differ by 1 , so imposed rule of cordial labeling is worked successfully. We also analyze the time complexity of the above algorithm which is super linear and it is $\bigcirc(n r)$. The time complexity of this algorithm is super linear and it is $\bigcirc(n r)$.

TABle 6 . Vertex label matrix of $K_{4,4} \times P_{4}$

$\begin{array}{llllllll}1 & 0 & 0 & 0 & 1 & 0 & 0 & 0 \\ 0 & 1 & 1 & 1 & 0 & 1 & 1 & 1 \\ 1 & 0 & 0 & 0 & 1 & 0 & 0 & 0 \\ 0 & 1 & 1 & 1 & 0 & 1 & 1 & 1\end{array}$

TABLE 7 . Edge label matrix of $K_{4,4} \times P_{4}$

$\begin{array}{lllllllllllllllllllllll}0 & 1 & 0 & 1 & 1 & 0 & 1 & 0 & 1 & 0 & 1 & 0 & 0 & 1 & 0 & 1 & 1 & 0 & 1 & 0 & 1 & 0 \\ 1 & 0 & 1 & 0 & 1 & 0 & 0 & 1 & 0 & 1 & 1 & 0 & 1 & 0 & 1 & 0 & 1 & 0 & 0 & 1 & 0 & 1 \\ 0 & 1 & 0 & 1 & 1 & 0 & 1 & 0 & 1 & 0 & 1 & 0 & 0 & 1 & 0 & 1 & 1 & 0 & 1 & 0 & 1 & 0 \\ 1 & 0 & 1 & 0 & 1 & 0 & 0 & 1 & 0 & 1 & 1 & 0 & 1 & 0 & 1 & 0 & 1 & 0 & 0 & 1 & 0 & 1\end{array}$




\section{Conclusion}

This is the first attempt to apply cordial labeling on the graph obtained by doing Cartesian product between bipartite graph and path. We have designed four algorithm to label the above said graph and all the above algorithms will work successfully. We have also analyze time complexity of the above algorithms and time complexity of the cordial labeling of the graph obtained by the Cartesian product between $K_{n, n} \times P_{r}$ i.e balanced bipartite and path is $\bigcirc(n r)$. A wide research area is open such type of graph structure, researcher can investigate this problem on the graph obtained by doing Cartesian product between complete bipartite graph with other simple graph.

Acknowledgement. The work is supported by the Department of Science and Technology, New Delhi, India, Ref. No.SB/S4/MS: 894/14.

\section{REFERENCES}

[1] Gallian, J.A., A dynamic survey of graph labeling, The Electronics Journal of Combinatorics, $17,(2010) 6$.

[2] Hegde, S.M., On Multiplicative Labelings of a Graph. Labeling of Discrete Structures and applications. Narosa Publishing House. New Delhi. 83-96 2008.

[3] Beineke, L.W., Hegde, S.M., Strongly Multiplicative graphs. Discuss.Math. Graph Theory, 21, (2001) 63-75.

[4] Cahit, I., Cordial graphs a weaker version of graceful and harmonious graphs. Ars Combin. 23, (1987) 201-207.

[5] Cahit, I., On cordial and 3-equitable labellings of graphs. Util. Math. 37, (1990) 189-198.

[6] Cahit, I., Status of graceful tree conjecture in 1989, in Topics in Combinatorics and Graph Theory. R. Bodendiek and R. Henn (eds). Physica-Verlag. Heidelberg. 1990.

[7] Cahit, I., Recent results and open problems on cordial graphs. Contemporary Methods in Graph Theory. R. Bodendiek (ed.). Wissenschaftsverlag.Mannheim. 209-230, 1990.

[8] Cahit, I., Equitable tree labellings. Ars Combin. 40, 279-286 (1995).

[9] Cahit, I., On harmonious tree labellings. Ars Combin. 41, (1995) 311-317.

[10] Cahit, I., H-cordial graphs. Bull. Inst. Combin. Appl. 18, (1996) 87-101.

[11] Cahit, I., Some totally modular cordial graphs. Discuss. Math. Graph Theory. 22, (2002) 247-258.

[12] Cahit, I., Graceful labelings of rooted complete trees. personal communication.

[13] Cahit, I., A note on graceful directed trees. personal communication.

[14] Cahit, I., Yilmaz, R., E3-cordial graphs. Ars Combin. 54, (2000) 119-127.

[15] Graham, R.L., Sloane, N.J.A., On additive bases an harmonious graphs. SIAM J. Algebr. Disc. Meth. 1(4), (1980) 382-404.

[16] Rosa, A., On certain valuations of the vertices of a graph. Thery of graphs. International Symposium, (1967) 349-355.

[17] Babujee, J.B., Shobana, L., Cordial Languages and Cordial Numbers. Journal of Applied Computer Science and Mathematics, 13(6), (2012) 9-12.

[18] Ulaganathan, P.P., Selvam, B., Vijaya, K.P., Signed Product Cordial Labeling in duplicate graphs of Bistar, Double Star and Triangular Ladder Graph. International Journal of Mathematics Trends and Technology(IJMTT), 33(1), (2016) 19-24.

[19] Santhi, M., Some Star and Bistar Related Signed Product Cordial Graphs. International Journal of Mathematical Archive, 6(10), (2015) 232-236. 
[20] Ho, Y.S., Lee, S.M., Shee, S.C., Cordial labelings of unicyclic graphs and generalized peterson graphs. Congr. Numer., 68, (1989) 109-122.

Current address: Sumonta Ghosh: Department of Mathematics, NIT Durgapur, W. Bengal, India-713209

E-mail address: mesumonta@gmail.com

ORCID Address: http://orcid.org/0000-0002-8198-8422

Current address: Anita Pal: Department of Mathematics, NIT Durgapur, W. Bengal, India713209

E-mail address: mesumonta@gmail.com

ORCID Address: http://orcid.org/0000-0002-2514-5463 